\title{
Uncertainty Principle and Bifurcations in the SU(2) Nonlinear Semiquantum Dynamics
}

\author{
Roberta Hansen1, Claudia M. Sarris ${ }^{1,2}$, Angelo Plastino ${ }^{3,4}$ \\ ${ }^{1}$ Department of Mathematics, Faculty of Engineering, University of Buenos Aires, Buenos Aires, Argentina \\ ${ }^{2}$ Common Basic Cycle, Chair of Physics, University of Buenos Aires, Buenos Aires, Argentina \\ ${ }^{3}$ Institute of Physics of La Plata, CCT-CONICET, National University of La Plata, La Plata, Argentina \\ ${ }^{4}$ Physics Department and IFISC-CSIC, University of the Balearic Island, Palma de Mallorca, Spain \\ Email:rhansen@fi.uba.ar,clsarris@fi.uba.ar, aplastino@gmail.com
}

How to cite this paper: Hansen, R., Sarris, C.M. and Plastino, A. (2018) Uncertainty Principle and Bifurcations in the SU(2) Nonlinear Semiquantum Dynamics. Applied Mathematics, 9, 1-16. https://doi.org/10.4236/am.2018.91001

Received: December 6, 2017

Accepted: January 8, 2018

Published: January 11, 2018

Copyright $\odot 2018$ by authors and Scientific Research Publishing Inc. This work is licensed under the Creative Commons Attribution International License (CC BY 4.0).

http://creativecommons.org/licenses/by/4.0/

\begin{abstract}
In this paper, a nonlinear semiquantum Hamiltonian associated to the special unitary group $\mathrm{SU}(2)$ Lie algebra is studied so as to analyze its dynamics. The treatment here applied allows for a reduction in: 1) the system's dimension, as well as 2) the number of system's parameters (to only three). We can now discern clear patterns in: 1) the complete characterization of the system's fixed points and 2) their stability. It is shown that the parameter associated to the uncertainty principle, which constitutes a very strong constraint, is the key one in determining the presence of fixed points and bifurcation curves in the parameter's space.
\end{abstract}

\section{Keywords}

Semiquantum Dynamics, Uncertainty Principle, Fixed Points, Bifurcation Curves

\section{Introduction}

Semiquantum Dynamics (SD) may be used to describe systems in which quantum and classical degrees of freedom coexist. One finds in [1] an exhaustive compilation of physical phenomena and technological applications successfully modeled by SD. It is also possible to encounter situations in which SD is used to describe physical phenomena [2] [3] [4] [5]. A nonlinear semiquantum Hamiltonian associated to the SU(2) Lie algebra is very useful to model the problem of quantum confinement, which is of interest for nanotechnology and solid state physics. In particular, if the quantum subsystem is associated to the SU(2) Lie algebra generators $\left\{\hat{\sigma}_{x}, \hat{\sigma}_{y}, \hat{\sigma}_{z}\right\}$, the uncertainty principle (UP) adopts a very 
simple form and turns out to be a motion invariant [6].

In [7], the authors consider the following semiquantum Hamiltonian

$$
\hat{H}=B \hat{\sigma}_{z}+C q \hat{\sigma}_{x}+\frac{p^{2}}{2 m}+D \frac{q^{4}}{4}-F \frac{q^{2}}{2},
$$

where $\hat{\sigma}_{x}$ and $\hat{\sigma}_{z}$ are quantum operators, the $x$ and $z$ components of a $1 / 2$ spin particle, while $q$ and $p$ are canonical conjugated classical variables (position and momentum) that obey the Hamilton equations [5] [8]. $B, C, m, D$, and $F$ are positive and constant parameters. The Hamiltonian given by Equation (1) represents a quantum $1 / 2$ spin particle interacting with an external magnetic field [2] [7] (due to the term $B \hat{\sigma}_{z}$ ). The particle is confined by the double well potential $V(q)=D \frac{q^{4}}{4}-F \frac{q^{2}}{2}$, generated by a classical particle of mass $m$ and it undergoes elastic reflections between the moving frontier, $\partial V$, of the double well potential. The term $\frac{p^{2}}{2 m}$ represents the classical kinetic energy. The classical and quantum variables are couple in non-linear fashion via the term, $C q \hat{\sigma}_{x}, C$ being the coupling constant. In [7], the authors concentrate on the likely presence of chaotic motion (semiquantum chaos) for special values of the coupling strength. The authors represent the trajectories for different initial conditions by stroboscopic plots, displaying regular and irregular dynamics. This Hamiltonian also may be reduced to the one in [2] (taking $F=0$ ). It also can be used to model the semiquantum differential equations of the spin-boson Hamiltonian of [9] (taking $D=0$ ). In [10], the authors considered the simplest case of a spin-boson Hamiltonian, i.e., a two level system coupled to one oscillator degree of freedom, and made a posterior semiclassical approximation, to obtain a semiquantum Hamiltonian similar to that given by Equation (1).

We consider that, since the Hamiltonian of Equation (1) is able to model the quantum confinement phenomenon, its dynamics deserves an exhaustive analysis. In the present work, we purport to give a full description of its phase space taking into account that, in conservative systems like this one, the motion is fully determined by the amount and disposition of its fixed points. We make a complete characterization of them and determine their stability according to the system's parameters values. In addition, we obtain the bifurcation curves which divide the phase space into the three different zones in which the fixed points are located, according to their stability. We present a dimensionless formulation, and because the uncertainty principle (UP) is an invariant of the motion for the nonlinear semiquantum Hamiltonians associated to the SU(2) Lie algebra [6] [11], we make a change of variables that removes it as external strong constrain to the system's motion equations. The UP provides then just an additional parameter in the concomitant new motion equations. We mention that, in [10], a similar method is used for a coupled quasiparticle-oscillator system, which enables the authors to study the existence of fixed points and bifurcation curves, allowing for a formulation in canonically conjugate variables of the excitonic 
subsystem. In our case, the change of variables offers some advantages which are highlighted in describing our treatment and summarized in the conclusions.

\section{Equations of Motion}

If we consider the generators of the $\mathrm{SU}(2)$ Lie algebra, $\left\{\hat{\sigma}_{x}, \hat{\sigma}_{y}, \hat{\sigma}_{z}\right\}$, it can be easily seen that they close a partial Lie algebra under commutation with the Hamiltonian of Equation (1), since the commutator of any $\hat{\sigma}_{i}$ with $\hat{H}$ may be expressed as a linear superposition of these generators. The semiquantum equations of motion are obtained through the Maximum Entropy Approach (MEP), using the MEP density operator $\hat{\rho}$ to evaluate the Hamiltonian's mean value, $\langle\hat{H}\rangle=\operatorname{Tr}(\hat{\rho} \hat{H})$ which, in turn, plays the role of a Hamilton function so as to obtain the (evolution) differential equations of motion corresponding to the classical degrees of freedom $q$ and $p$ (the prescription given by the MEP, in order to find the density operator, is a standard procedure. The interested reader can consult [6] [12] [13] [14] to become familiar with the subject). The MEP density operator $\hat{\rho}$ corresponds to a non-pure state given that it is constructed from a set of noncommuting observable $\left\{\hat{\sigma}_{x}, \hat{\sigma}_{y}, \hat{\sigma}_{z}\right\}$ (the generators of the $\mathrm{SU}(2)$ Lie algebra).

Following the prescription given in [6] [12] [13], we obtain the equations of motion for the system given by Equation (1):

$$
\begin{aligned}
& \frac{\mathrm{d}\left\langle\hat{\sigma}_{x}\right\rangle}{\mathrm{d} t}=-2 B\left\langle\hat{\sigma}_{y}\right\rangle, \\
& \frac{\mathrm{d}\left\langle\hat{\sigma}_{y}\right\rangle}{\mathrm{d} t}=2 B\left\langle\hat{\sigma}_{x}\right\rangle-2 C q\left\langle\hat{\sigma}_{z}\right\rangle, \\
& \frac{\mathrm{d}\left\langle\hat{\sigma}_{z}\right\rangle}{\mathrm{d} t}=2 C q\left\langle\hat{\sigma}_{y}\right\rangle, \\
& \frac{\mathrm{d} q}{\mathrm{~d} t}=\frac{p}{m}, \\
& \frac{\mathrm{d} p}{\mathrm{~d} t}=C\left\langle\hat{\sigma}_{x}\right\rangle-D q^{3}+F q,
\end{aligned}
$$

and they must obey the uncertainty relation [6] [11] which, for the SU(2) Lie algebra case, adopts the form [6]:

$$
0<\langle\hat{\sigma}\rangle^{2}=\left\langle\hat{\sigma}_{x}\right\rangle^{2}+\left\langle\hat{\sigma}_{y}\right\rangle^{2}+\left\langle\hat{\sigma}_{z}\right\rangle^{2}<1 .
$$

We will consider the whole range of values that the polarization vector $\langle\hat{\sigma}\rangle$ can achieve in the interval $(0,1)$, given that the generators of the $\mathrm{SU}(2)$ Lie algebra $\left\{\hat{\sigma}_{x}, \hat{\sigma}_{y}, \hat{\sigma}_{z}\right\}$ constitute a complete set of noncommuting observables. Thus, we are dealing with a non pure quantum state $\hat{\rho}$. Equation (7) defines the well-known Bloch sphere, whose "radius", $\langle\hat{\sigma}\rangle$, remains a constant of the motion while its possible values $0<\langle\hat{\sigma}\rangle<1$ are determined by the initial conditions imposed on Equations (2)-(6). It is also taken into account that the system's energy (evaluated via the non pure state density operator $\hat{\rho}$ ) [6] [13]: 


$$
\langle\hat{H}\rangle=B\left\langle\hat{\sigma}_{z}\right\rangle+C q\left\langle\hat{\sigma}_{x}\right\rangle+\frac{p^{2}}{2 m}+D \frac{q^{4}}{4}-F \frac{q^{2}}{2},
$$

must remain a constant of motion during the whole temporal evolution [6].

In order to find the fixed points it is convenient to express Equations (2)-(6), (7), and (8) in dimensionless form [15], by defining new variables, $\tau, x, y, z, q$ and $p$ :

$$
\begin{aligned}
& \left\langle\hat{\sigma}_{x}\right\rangle=\alpha x, \quad\left\langle\hat{\sigma}_{z}\right\rangle=\delta z,\left\langle\hat{\sigma}_{y}\right\rangle=\gamma y, \\
& t=T_{0} \tau, \quad q=\mu q, \quad p=v p,
\end{aligned}
$$

with

$$
\begin{aligned}
& \alpha=\gamma=\delta=\frac{D B^{3}}{C^{4}}, T_{0}=\frac{1}{2 B}, \mu=\frac{B}{C}, \\
& \nu=\frac{D B^{2}}{2 C^{3}}, \quad \varepsilon=\frac{D}{4 m C}, \quad s=\frac{F C^{2}}{D B^{2}} .
\end{aligned}
$$

Accordingly, Equations (2)-(6) and (7) become:

$$
\begin{aligned}
& \frac{\mathrm{d} x}{\mathrm{~d} \tau}=-y, \\
& \frac{\mathrm{d} y}{\mathrm{~d} \tau}=x-q z, \\
& \frac{\mathrm{d} z}{\mathrm{~d} \tau}=q y, \\
& \frac{\mathrm{d} q}{\mathrm{~d} \tau}=\varepsilon p, \\
& \frac{\mathrm{d} p}{\mathrm{~d} \tau}=-x-q^{3}+s q, \\
& x^{2}+y^{2}+z^{2}=r^{2}=(\langle\hat{\sigma}\rangle / \alpha)^{2},
\end{aligned}
$$

and from Equation (8) the system's energy reads:

$$
\langle h\rangle=\frac{\langle\hat{H}\rangle}{B \alpha}=z+q x+\varepsilon \frac{p^{2}}{2}+\frac{q^{4}}{4}-s \frac{q^{2}}{2} .
$$

\section{The System's Fixed Points}

In order to determine the system's fixed points we proceed, as usual, by equating (11)-(15) to zero. Note that the existence of them does not depend on the parameter $\varepsilon$, but only on the values of $r$ and $s$. From Equation (13), there exist two situations: the cases $q^{*}=0$ and $q^{*} \neq 0$.

\subsection{Case $q^{*}=0$}

From $\frac{\mathrm{d} x}{\mathrm{~d} \tau}=0$ in Equation (11), it follows $y=0$. From $\frac{\mathrm{d} y}{\mathrm{~d} \tau}=0$ in Equation (12), it follows $x=0$. So, from Equation (16), we are led to $z= \pm r$. From 
$\frac{\mathrm{d} q}{\mathrm{~d} \tau}=0$ in Equation (14), it follows $p=0$. Therefore, in this case, one obtains two fixed points, $\left[x^{*}, y^{*}, z^{*}, q^{*}, p^{*}\right]=[0,0, \pm r, 0,0]$, where $r=\langle\hat{\sigma}\rangle / \alpha$, $\mathrm{PN}=[0,0, r]$ and $\mathrm{PS}=[0,0,-r]$ being the north and south pole of the dimensionless Bloch sphere.

\subsection{Case $q^{*} \neq 0$}

From Equations (11)-(15) it follows that these kinds of fixed points must fulfill:

$$
y^{*}=0, p^{*}=0, x^{*}=q^{*}\left(s-q^{* 2}\right) \text { and } z^{*}=s-q^{* 2},
$$

so they adopt the appearance $\left[q^{*}\left(s-q^{* 2}\right), 0, s-q^{* 2}, q^{*}, 0\right]$, and must obey the strong constraint given by Equation (16), the uncertainty principle, which in terms of $q^{*}$, reads:

$$
\left(s-q^{* 2}\right)^{2}\left(1+q^{* 2}\right)-r^{2}=0
$$

with $r=\langle\hat{\sigma}\rangle / \alpha, 0<\langle\hat{\sigma}\rangle<1$. Thus, $q^{*}=q^{*}(r, s)$ are the roots of Equation (19), which should be tackled numerically. The system's phase space, given by Equations (11)-(15), is five-dimensional. However, the Jacobian matrix at the fixed point must have, at least, one null eigenvalue, since the uncertainty condition of Equation (16) is an external constraint added to the equations of motion. This means that, in fact, the solutions lie on a $4 \mathrm{D}$ invariant manifold, $\mathcal{M}$.

Accordingly, we represent the quantum degrees of freedom, $[x, y, z]$, in spherical coordinates:

$$
x=r \cos (\theta) \sin (\varphi), \quad y=r \sin (\theta) \sin (\varphi), z=r \cos (\varphi),
$$

with $0 \leq \theta<2 \pi, 0<\varphi<\pi, \quad r=\langle\hat{\sigma}\rangle / \alpha$ and we study the system's fixed points by means of the four-dimensional variables, $\xi=\left[\xi_{1}, \xi_{2}, \xi_{3}, \xi_{4}\right]=[\theta, \varphi, q, p]$.

Using the relations:

$$
z^{2}=r^{2} \cos ^{2}(\varphi), \quad \tan (\theta)=\frac{y}{x}
$$

we find:

$$
\dot{\theta}=\frac{\cos ^{2}(\theta)}{x^{2}}(\dot{y} x-y \dot{x}), \quad \dot{\varphi}=-\frac{z \dot{z}}{r^{2} \sin (\varphi) \cos (\varphi)} .
$$

The system of Equations (11)-(16) becomes now:

$$
\begin{aligned}
& \dot{\theta}=1-q \cos (\theta) \frac{\cos (\varphi)}{\sin (\varphi)}, \\
& \dot{\varphi}=-q \sin (\theta), \\
& \dot{q}=\varepsilon p, \\
& \dot{p}=-r \cos (\theta) \sin (\varphi)-q^{3}+s q,
\end{aligned}
$$

where () means $\frac{\mathrm{d}}{\mathrm{d} \tau}()$, and $0<(\alpha r)^{2}=\langle\hat{\sigma}\rangle^{2}<1$. The system's energy given 
by Equation (17) becomes:

$$
\langle h\rangle=r(\cos (\varphi)+q \sin (\varphi) \cos (\theta))+\varepsilon \frac{p^{2}}{2}+\frac{q^{4}}{4}-s \frac{q^{2}}{2} .
$$

We claim that this change of variables (CV) offers some advantages:

1) The quantum variables $\theta$ and $\varphi$ obey the relationship:

$$
\dot{\varphi}=\frac{1}{r \sin (\varphi)} \frac{\partial\langle h\rangle}{\partial \theta}, \quad \dot{\theta}=-\frac{1}{r \sin (\varphi)} \frac{\partial\langle h\rangle}{\partial \varphi}
$$

as if they were canonical spherical-conjugates, meanwhile the classical ones, $q$ and $p$, obey, as usual, the Hamilton's equations [6] [13]:

$$
\dot{q}=\frac{\partial\langle h\rangle}{\partial p}, \quad \dot{p}=-\frac{\partial\langle h\rangle}{\partial q} .
$$

Thus, the fixed points $\xi^{*}$ of the system given by Equations (23)-(26), are the critical points of the energy function $\langle h\rangle$ of Equation (27), since:

$$
\frac{\partial\langle h\rangle}{\partial \xi_{i}}\left(\xi^{*}\right)=0, \quad i=1,2,3,4
$$

2) The uncertainty relation in Equation (16), now of the form $0<(r \alpha)^{2}=\langle\hat{\sigma}\rangle^{2}<1$, is incorporated into the new system's equations in a natural way by reducing the system dimension and removing a superfluous null eigenvalue. One may speak of linearization of the original system at each fixed point.

3) Our CV provides a better characterization of the fixed points, since with this change we find a generic expression for them explicitly written in terms of the variable $q$. This fact facilitates the study of 1 ) the presence of bifurcations curves in the parameter's space, and 2) the stability analysis of the fixed points.

To obtain the fixed points, $\xi^{*}=\left[\theta^{*}, \varphi^{*}, q^{*}, p^{*}\right]$, we equate (23)-(26) to zero. From $\dot{q}=0$ in Equation (25), it follows that $p^{*}=0$, and from $\dot{\varphi}=0$ in Equation (24), we are led to $\theta_{0}^{*}=0+2 k \pi$ or $\theta_{\pi}^{*}=\pi+2 k \pi, k \in \mathbb{Z}$.

\subsubsection{Case $\theta_{0}^{*}=0+2 k \pi$}

From $\dot{\theta}=0$ in Equation (23) and $\cos \left(\theta^{*}\right)=1$, it follows that:

$$
0=1-q^{*} \cos \left(\theta^{*}\right) \frac{\cos \left(\varphi^{*}\right)}{\sin \left(\varphi^{*}\right)}=1-\frac{q^{*}}{\tan \left(\varphi^{*}\right)},
$$

so that:

$$
q^{*}=\tan \left(\varphi^{*}\right)
$$

Then, in this case, $\xi^{*}$ adopts the generic form $\xi_{0}^{*}=\left[\theta_{0}^{*}, \arctan \left(q_{0}^{*}\right), q_{0}^{*}, 0\right]$. From Equation (32), $q^{* 2}=\frac{\sin ^{2}\left(\varphi^{*}\right)}{\cos ^{2}\left(\varphi^{*}\right)}$, and $\sin ^{2}\left(\varphi^{*}\right)=\frac{q^{* 2}}{1+q^{* 2}}$. Since $\sin (\varphi)>0$, then: 


$$
\sin \left(\varphi^{*}\right)=\frac{\left|q^{*}\right|}{\sqrt{1+q^{* 2}}} .
$$

From $\dot{p}=0$ in Equation (26) and $\cos \left(\theta^{*}\right)=1$ one has:

$$
0=-r \cos \left(\theta^{*}\right) \sin \left(\varphi^{*}\right)-q^{* 3}+s q^{*}=-r \sin \left(\varphi^{*}\right)+q^{*}\left(s-q^{* 2}\right),
$$

and then:

$$
r \sin \left(\varphi^{*}\right)=q^{*}\left(s-q^{* 2}\right) .
$$

Replacing Equation (33) into Equation (35), it follows that $q_{0}^{*}$ must be the solution of the following equation:

$$
q\left(s-q^{2}\right)=\frac{r|q|}{\sqrt{1+q^{2}}} .
$$

Since $q^{*} \neq 0$, the right side on Equation (36) is positive. Thus, the possible values of $q_{0}^{*}$ are restricted to the range $(-\infty,-\sqrt{s}) \cup(0, \sqrt{s})$. In each case, the amount of solutions will be obtained graphically by means of the intersection points of two curves, namely:

$$
f(q)=\left\{\begin{array}{ll}
q^{2}-s, & q<0 \\
s-q^{2}, & q>0
\end{array} \text { and } \quad g(q)=\frac{r}{\sqrt{1+q^{2}}} .\right.
$$

- $q<0$. Figure 1 depicts the intersection between the left branch of $f(q)$ and $g(q)$. It is possible to see that for all $r, s>0$, there is always a unique solution, $q_{0_{1}}^{*}=q_{0_{1}}^{*}(r, s)$, in the range of interest, and so a corresponding fixed point, $\xi_{0_{1}}^{*}=\left[0, \arctan \left(q_{0_{1}}^{*}\right), q_{0_{1}}^{*}, 0\right]$, is obtained. Note also that this point does not bifurcate in the $(r, s)$-parameter space.

- $q>0$. Here, we are looking for the intersection points of the right branch of $f(q)$ and $g(q)$. The situations for different values of $r$ and $s$ are depicted in Figure 2, by considering $r$ as a "fixed" parameter and $s$ going down from $s>r$ to $s<r$.

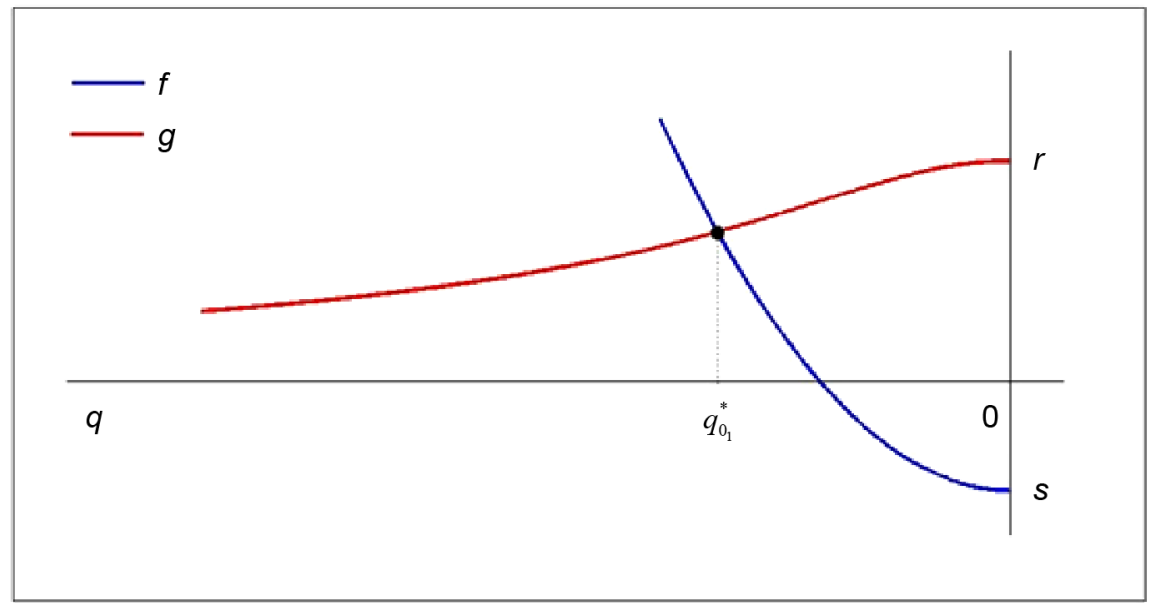

Figure 1. The graph of the functions $g$ and the left branch of $f$ of Equation (37) for $r, s>0$, showing the unique intersection point $q_{0_{1}}^{*}$ existing for $q<0$. 


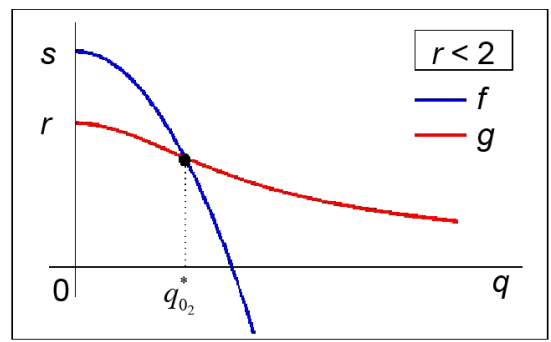

(a)

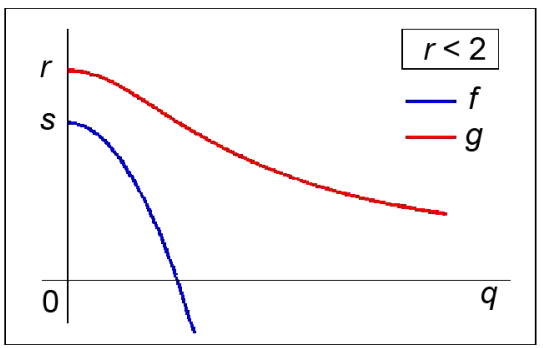

(c)

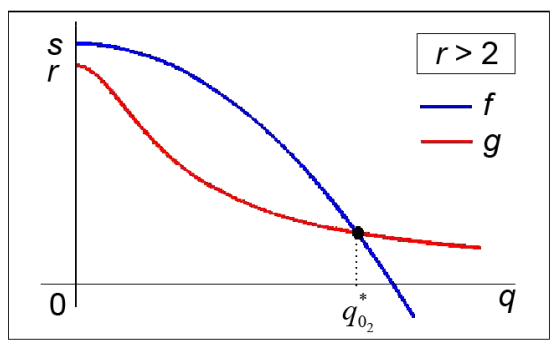

(b)

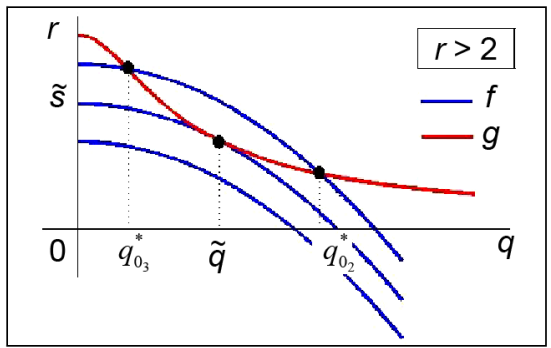

(d)

Figure 2. The graph of the functions $g$ and the right branch of $f$ of Equation (37) for $r, s>0$. The figures in the first column ((a)-(c)) depict the case where $f$ is more convex than $g$ at $q=0 \quad(r<2)$; in the second column ((b)-(d)), $g$ is more convex than $f$ at $q=0 \quad(r>2)$. (a) and (b) show the unique intersection point $q_{0_{2}}^{*}$ existing for $q>0$, when $r<s$. (c) there is no intersection point when $s<r$. (d) there are two intersection points, $q_{0,2}^{*}$ and $q_{0,3}^{*}$, when $\tilde{s}<s<r, \tilde{s}$ being a critical value for which $f$ and $g$ become tangent. When $s<\tilde{s}$ the graphs do not intersect at all.

For $s>r$, Figure 2(a) and Figure 2(b) show that the two curves intersect at one point at $q_{0_{2}}^{*}=q_{0_{2}}^{*}(r, s)$, this being the unique solution of Equation (36) in the range of interest, adding a new fixed point, $\xi_{0_{2}}^{*}$.

Decreasing the $s$ value, the curves become tangent at $q=0$, at the critical value $s=r$. Note that $s$ is a "rigid" parameter since only produces $f$ graph shifts, in contrast to the "flexible" parameter $r$ which bends the graph of $g$. The scenario splits into two ones when $s<r$, depending on the convexity of $g$ as compared to the $f$ value at $q=0$, i.e., depending on the absolute values of the second derivatives:

$$
\left|f^{\prime \prime}(0)\right|=2 \text { and } \quad\left|g^{\prime \prime}(0)\right|=\left|\frac{-r\left(1-2 q^{2}\right)}{\sqrt{\left(1+q^{2}\right)^{5}}}\right|_{q=0} \mid=r .
$$

Thus, for $s<r<2 \quad\left(\left|g^{\prime \prime}(0)\right|<\left|f^{\prime \prime}(0)\right|\right.$, see Figure $\left.2(\mathrm{c})\right)$, there is no intersection point between $f$ and $g$. However, for $s<r$ but $r>2$ $\left(\left|g^{\prime \prime}(0)\right|>\left|f^{\prime \prime}(0)\right|\right.$, see Figure 2(d)), the graphs intersect at a new point, $q_{0_{3}}^{*}=q_{0_{3}}^{*}(r, s)$, giving rise to another solution in the range of interest. This fact makes the line $s=r$ to be a codimension-1 bifurcation curve in the $(r, s)$ -parameter space: an imperfect saddle-node bifurcation occurs for $r<2$, since the fixed point $\xi_{0_{2}}^{*}$ is lost, and another imperfect saddle-node bifurcation 
occurs for $r>2$, since a new fixed point, $\xi_{0_{3}}^{*}$, is created (the term "imperfect" means that only one point is created/extinguished, instead of two, as would happen in a "perfect" saddle-node. This is due to the loss of the quadratic symmetry of $s-q^{2}$ when considering only its right branch $(q>0)$ [15].

This last scenario $(r>2)$ persists until the two graphs becomes tangent again at the critical value $\tilde{s}=\tilde{s}(t)$, when $q_{0_{2}}^{*}$ and $q_{0_{3}}^{*}$ meet each other at $\tilde{q}=\tilde{q}(r, s)$, with $(\tilde{q}, f(\tilde{q}))=(\tilde{q}, g(\tilde{q}))$ the tangency point. For $s$ below this critical value $\tilde{s}$, the two graphs do not match any longer, and the fixed points $\xi_{0_{2}}^{*}$ and $\xi_{0_{3}}^{*}$ are mutually destroyed. The system undergoes a ("perfect") saddle-node bifurcation at $\tilde{s}$, and this makes the relation $s=s(r)$ to be another codimension-1 bifurcation curve in the $(r, s)$-parameter space. Note the fundamental role that plays the uncertainty principle parameter $r$ in the coming into being of these bifurcation curves.

To find the relation $s=s(r)$ at $\tilde{q}$, we proceed by equating the first derivatives:

$$
f^{\prime}(q)=-2 q \quad \text { and } \quad g^{\prime}(q)=\frac{-r q}{\sqrt{\left(1+q^{2}\right)^{3}}} .
$$

From this, we obtain the relation between $\tilde{q}$ and $r$.

$$
\tilde{q}=\tilde{q}(r)=\sqrt{\sqrt[3]{\frac{r^{2}}{4}}-1},
$$

valid for $r>2$. Evaluating $f$ and $g$ at Equation (40), and so equating $f(\tilde{q})$ to $g(\tilde{q})$, we find the relation between $r$ and $s$.

$$
r=r(s)=2 \sqrt{\left(\frac{s+1}{3}\right)^{3}},
$$

or, equivalently,

$$
s=s(r)=3 \sqrt[3]{\frac{r^{2}}{4}}-1 .
$$

Replacing Equation (41) into Equation (40), we find the relation between $\tilde{q}$ and $\tilde{s}$, that allows to better fit the possible ranges for $q_{0_{2}}^{*}$ and $q_{0_{3}}^{*}$, within the range $(0, \sqrt{s})$, which will be useful later on:

$$
\tilde{s}=3 \tilde{q}^{2}+2,
$$

or, equivalently:

$$
\tilde{q}=\sqrt{\frac{\tilde{s}-2}{3}} .
$$

Thus, as $q_{0_{3}}^{*}<\tilde{q}<q_{0_{2}}^{*}$ (Figure 2(d)), we have for $s<r$ :

$$
q_{0_{3}}^{*} \in\left(0, \sqrt{\frac{s-2}{3}}\right) \text { and } q_{0_{2}}^{*} \in\left(\sqrt{\frac{s-2}{3}}, \sqrt{s}\right) \text {. }
$$

Note that the bifurcation curve $s(r)$ meets the line $s=r$ tangentially at a 
codimension-2 point $(2,2)$ in the $(r, s)$-parameter space (Figure 3$)$.

\subsubsection{Case $\theta_{\pi}^{*}=\pi+2 k \pi$}

From $\dot{\theta}=0$ in Equation (23) and $\cos \left(\theta^{*}\right)=-1$, it follows that:

$$
q^{*}=-\tan \left(\varphi^{*}\right) \text {. }
$$

Accordingly, in this case $\xi^{*}$ adopts the generic form $\xi_{\pi}^{*}=\left[\theta_{\pi}^{*},-\arctan \left(q_{\pi}^{*}\right), q_{\pi}^{*}, 0\right]$. Equation (33) is still valid, but from $\dot{p}=0$ in Equation (26) and $\cos \left(\theta^{*}\right)=-1$, one has:

$$
r \sin \left(\varphi^{*}\right)=q^{*}\left(q^{*}-s\right)
$$

Replacing (33) into (47), it follows that $q_{\pi}^{*}$ must be the solutions of the equation:

$$
q\left(q^{2}-s\right)=\frac{r|q|}{\sqrt{1+q^{2}}} .
$$

Accordingly, in this case the possible values of $q_{\pi}^{*}$ are restricted to the range $(-\sqrt{s}, 0) \cup(\sqrt{s},+\infty)$. Therefore, the solutions should now be obtained by the intersection points of the functions $-f(q)$ and $g(q)$ (defined in (37)) according to the $r$ and s values. It is easy to see that, for symmetry reasons, the analysis is completely analogous to the one developed in the previous Section. Then, the solutions thus obtained are: $q_{\pi_{1}}^{*}=-q_{0_{1}}^{*} \in(\sqrt{s},+\infty)$ for all $r, s>0$,

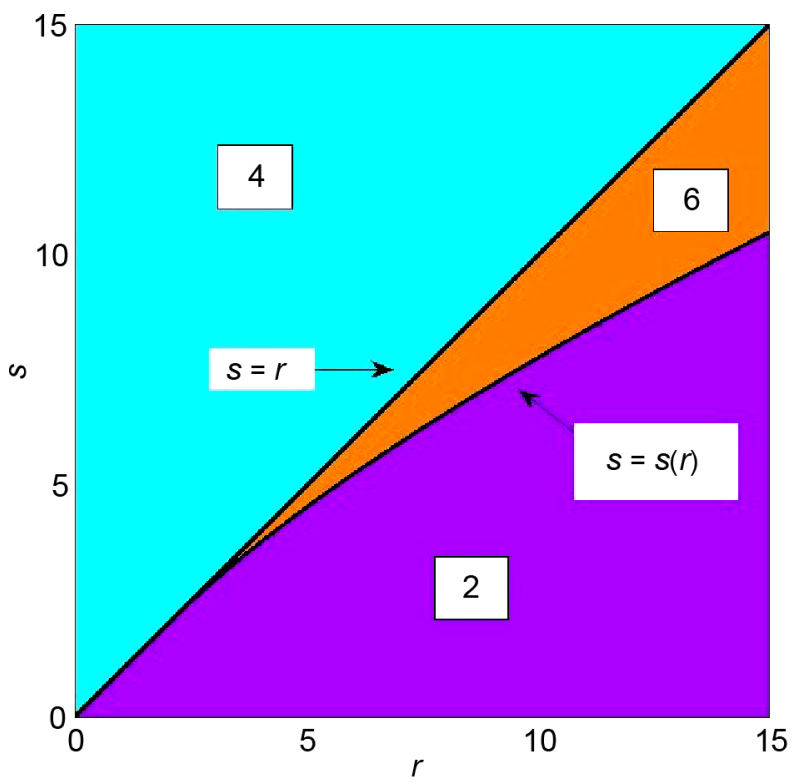

Figure 3. The $(r, s)$-parameter space and the codimension-1 bifurcation curves: the $s=r$ and $s=s(r)=3 \sqrt[3]{\frac{r^{2}}{4}}-1$ for $r>2$, that meet tangentially at the codimension-2 point $(r ; s)=(2,2)$. The number labeling each region indicates the quantity of non-degenerate fixed points. Cyan: $\xi_{0_{1}}^{*}, \xi_{0_{2}}^{*}, \xi_{\pi_{1}}^{*}, \xi_{\pi_{2}}^{*}$; orange: $\xi_{0_{1}}^{*}, \xi_{0_{2}}^{*}, \xi_{0_{3}}^{*}, \xi_{\pi_{1}}^{*}$, $\xi_{\pi_{2}}^{*}, \xi_{\pi_{3}}^{*} ;$ violet: $\xi_{0_{1}}^{*}, \xi_{\pi_{1}}^{*}$. 
$q_{\pi_{2}}^{*}=-q_{0_{2}}^{*} \in\left(-\sqrt{s},-\sqrt{\frac{s-2}{3}}\right)$ for $s>r, q_{\pi_{3}}^{*}=-q_{0_{3}}^{*} \in\left(-\sqrt{\frac{s-2}{3}}, 0\right)$ for

$s(r)<s<r, r>2$, and the same bifurcation curves, $s=r$ and $s=s(r)$, are found. Note also that $\varphi_{0}^{*}=\varphi_{\pi}^{*}$.

Summing up, the amount of fixed points in the $(r, s)$-plane is (Figure 3$)$ :

- $r<s \rightarrow \xi_{0_{1}}^{*}, \xi_{\pi_{1}}^{*}, \xi_{0_{2}}^{*}, \xi_{\pi_{2}}^{*}$,

- $s \leq r\left\{\begin{array}{l}s \leq \max \{2, s(r)\} \rightarrow \xi_{0_{1}}^{*}, \xi_{\pi_{1}}^{*}, \\ s \geq \max \{2, s(r)\} \rightarrow \xi_{0_{1}}^{*}, \xi_{\pi_{1}}^{*}, \xi_{0_{2}}^{*}, \xi_{\pi_{2}}^{*}, \xi_{0_{3}}^{*}, \xi_{\pi_{3}}^{*}\end{array}\right.$

\section{The Stability of the Fixed Points}

The system given by Equation (1) (and then by Equation (27)) is conservative. Thus, the local behavior at the fixed point may be studied by considering the energy $\langle h\rangle$ of Equation (27) as a function of $\xi=[\theta, \varphi, q, p],\langle h\rangle: \mathcal{M} \rightarrow \mathbb{R}$, which is a constant of motion, since $\langle\dot{h}\rangle=0$. The $h$-level sets, $\langle h\rangle(\xi)=h$, are $3 \mathrm{D}$ invariant manifolds containing orbits given by the equations of motion. The $h$-level sets help in understanding the structure of the system's phase space. The local behavior around a fixed point will be determined by the index $k$ of $\xi^{*}$ as a non-degenerate critical point of the Morse function $\langle h\rangle$ [16].

Definition 1. The instability index $k=k\left(\xi^{*}\right)$ is the number of negative eigenvalues of the Hessian matrix of $\langle h\rangle$ at $\xi^{*}, H_{\xi^{*}}\langle h\rangle=\left[\frac{\partial^{2}\langle h\rangle}{\partial \xi_{i} \partial \xi_{j}}\right]\left(\xi^{*}\right)$ (whenever $\xi^{*}$ is a non-degenerate critical point, i.e. $\operatorname{det}\left(H_{\xi^{*}}\langle h\rangle\right) \neq 0$ ).

This is to say, $k$ is the number of independent directions along which $\langle h\rangle$ decrease from $\xi^{*}$. Therefore, if $k\left(\xi^{*}\right)=0$, the $h$-level set around $\xi^{*}$, corresponds to a positive definite quadratic form, $\xi^{*}$ is a local minimum of $\langle h\rangle$ and a system's nonlinear center. If, in our case, $0<k\left(\xi^{*}\right)<4, \xi^{*}$ it is unstable, and of the saddle type.

\subsection{Obtaining the Index $k$ of $\xi^{*}$}

From Equation (27) the Hessian matrix of $\langle h\rangle$ at $\xi^{*}$ is:

$$
\begin{aligned}
& H_{\xi^{*}}\langle h\rangle=\left[\begin{array}{cccc}
\langle h\rangle_{\theta \theta}^{*} & 0 & 0 & 0 \\
0 & \langle h\rangle_{\varphi \varphi}^{*} & \langle h\rangle_{\varphi q}^{*} & 0 \\
0 & \langle h\rangle_{q \varphi}^{*} & \langle h\rangle_{q q}^{*} & 0 \\
0 & 0 & 0 & \langle h\rangle_{p p}^{*}
\end{array}\right] \\
& =\left[\begin{array}{cccc}
-r q^{*} \cos \left(\theta^{*}\right) \sin \left(\varphi^{*}\right) & 0 & 0 & 0 \\
0 & -r \cos \left(\varphi^{*}\right)-r q^{*} \cos \left(\theta^{*}\right) \sin \left(\varphi^{*}\right) & r \cos \left(\theta^{*}\right) \cos \left(\varphi^{*}\right) & 0 \\
0 & r \cos \left(\theta^{*}\right) \cos \left(\varphi^{*}\right) & 3 q^{* 2}-s & 0 \\
0 & 0 & 0 & \varepsilon
\end{array}\right]
\end{aligned}
$$

Since $H_{\xi^{*}}\langle h\rangle$ is a square-block diagonal matrix, its eigenvalues are those 
from the block submatrices. Taking into account that for a $2 \times 2$-matrix the eigenvalues may be written in terms of its trace and determinant, we have, then, that the four eigenvalues of $H_{\xi^{*}}\langle h\rangle$ are:

$$
\lambda_{1}\left(\xi^{*}\right)=\langle h\rangle_{\theta \theta}^{*}, \lambda_{2,3}\left(\xi^{*}\right)=\frac{t r \pm \sqrt{t r^{2}-4 \Delta}}{2}, \text { and } \lambda_{4}\left(\xi^{*}\right)=\langle h\rangle_{p p}^{*},
$$

where $\operatorname{tr}=\langle h\rangle_{\varphi \varphi}^{*}+\langle h\rangle_{q q}^{*}$ and $\Delta=\langle h\rangle_{\varphi \varphi}^{*}\langle h\rangle_{q q}^{*}-\langle h\rangle_{q \varphi}^{* 2}$, are the trace and determinant of the block $\left[\begin{array}{c}\langle h\rangle_{\varphi \varphi}^{*}\langle h\rangle_{\varphi q}^{*} \\ \langle h\rangle_{q \varphi}^{*}\langle h\rangle_{q q}^{*}\end{array}\right]$, respectively. The instability index is, then, $k\left(\xi^{*}\right)=\#\left\{\lambda\left(\xi^{*}\right)<0\right\}$.

From Equation (26), $r \cos \left(\theta^{*}\right) \sin \left(\varphi^{*}\right)=s q^{*}-q^{* 3}$, from Equation (32), $q^{*} \cos \left(\varphi^{*}\right)=\sin \left(\varphi^{*}\right)$ for $\theta_{0}^{*}$, and from Equation (46), $q^{*} \cos \left(\varphi^{*}\right)=-\sin \left(\varphi^{*}\right)$ for $\theta_{\pi}^{*}$. Therefore, the matrix in Equation (49) for $\xi_{0}^{*}$, is thus expressed in terms of $q^{*}$ :

$$
H_{\xi_{0}^{*}}\langle h\rangle=\left[\begin{array}{cccc}
q^{*^{2}}\left(q^{*^{2}}-s\right) & 0 & 0 & 0 \\
0 & \left(q^{*^{2}}-s\right)\left(1+q^{*^{2}}\right) & s-q^{*^{2}} & 0 \\
0 & s-q^{*^{2}} & 3 q^{*^{2}}-s & 0 \\
0 & 0 & 0 & \varepsilon
\end{array}\right],
$$

and for $\xi_{\pi}^{*}, H_{\xi_{\pi}^{*}}\langle h\rangle$ is the same except that $\langle h\rangle_{\varphi q}^{*}=\langle h\rangle_{q \varphi}^{*}=q^{* 2}-s$.

The eigenvalues $\lambda_{i}\left(\xi^{*}\right)$ of Equation (50) in terms of $q^{*}$ read:

$$
\lambda_{4}\left(\xi^{*}\right)=\varepsilon>0, \text { for all } \xi^{*},
$$

guaranteeing $k\left(\xi^{*}\right)<4$ for all $\xi^{*}$, as befits to a conservative system which cannot posses a completely unstable critical point.

$$
\lambda_{1}\left(\xi^{*}\right)=q^{*^{2}}\left(q^{*^{2}}-s\right) \begin{cases}>0, & \left|q^{*}\right| \in(\sqrt{s},+\infty) \rightarrow \xi_{0_{1}, \pi_{1}}^{*}, \\ <0, & \left|q^{*}\right| \in(0, \sqrt{s}) \rightarrow \boldsymbol{\xi}_{0_{2}, \pi_{2}}^{*}, \xi_{0_{3}, \pi_{3}}^{*} .\end{cases}
$$

Note that $\lambda_{1}\left(\xi^{*}\right) \neq 0$ because $q^{*} \neq 0$, and also, $q^{* 2} \neq s$ from Equation (19). For $\lambda_{2,3}\left(\xi^{*}\right)$ :

$$
\begin{aligned}
\Delta & =\lambda_{2}\left(\xi^{*}\right) \cdot \lambda_{3}\left(\xi^{*}\right)=\left(q^{* 2}-s\right)\left(1+q^{* 2}\right)\left(3 q^{* 2}-s\right)-\left(s-q^{* 2}\right)^{2} \\
& =q^{* 2}\left(q^{* 2}-s\right)\left(3 q^{* 2}+2-s\right),
\end{aligned}
$$

and using Equation (45), it follows that

$$
=\left\{\begin{array}{l}
s<2\left\{\begin{array}{l}
>0,\left|q^{*}\right| \in(\sqrt{s},+\infty) \rightarrow \xi_{0_{1}, \pi_{1}}^{*} \\
<0,\left|q^{*}\right| \in(\sqrt{s}, 0) \rightarrow \xi_{0_{2}, \pi_{2}}^{*}
\end{array}\right. \\
s>2\left\{\begin{array}{l}
>0,\left|q^{*}\right| \in\left(0, \sqrt{\frac{s-2}{3}}\right) \cup(\sqrt{s},+\infty) \rightarrow \xi_{0_{1}, \pi_{1}}^{*}, \xi_{0_{3}, \pi_{3}}^{*} \\
<0,\left|q^{*}\right| \in\left(\sqrt{\frac{s-2}{3}}, \sqrt{s}\right) \rightarrow \xi_{0_{2}, \pi_{2}}^{*}
\end{array}\right.
\end{array}\right.
$$


From Equation (50), if $\Delta<0$, it follows that $\lambda_{2}>0$ and $\lambda_{3}<0$. Thus, for $\xi_{0_{2}, \pi_{2}}^{*}$, we can just conclude, from Equations (52)-(54), that $k\left(\xi_{0_{2}, \pi_{2}}^{*}\right)=2$ For the remaining critical points, things depends on the sign of $t r$ in the corresponding intervals:

$$
\begin{aligned}
& \operatorname{tr}=\left(q^{* 2}-s\right)\left(1+q^{* 2}\right)+3 q^{* 2}-s \\
& = \begin{cases}>0 & \left|q^{*}\right| \in(\sqrt{s},+\infty) \rightarrow \xi_{0_{1}, \pi_{1}}^{*} \\
<0 & \left|q^{*}\right| \in\left(0, \sqrt{\frac{s-2}{3}}\right) \rightarrow \xi_{0_{3}, \pi_{3}}^{*} \quad(s>2)\end{cases}
\end{aligned}
$$

Then, from Equations (50), (54) and (55), $\lambda_{2,3}\left(\xi_{0_{1}, \pi_{1}}^{*}\right)>0, \lambda_{2,3}\left(\xi_{0_{3}, \pi_{3}}^{*}\right)<0$, and therefore, from Equations (52)-(55), it follows that $k\left(\xi_{0_{1}, \pi_{1}}^{*}\right)=0$ (nonlinear centers), and $k\left(\xi_{0_{3}, \pi_{3}}^{*}\right)=3$. The summary of results is displayed in Table 1 .

Figure 4 and Figure 5 illustrate a case relative to the orange zone in Figure 3 , for which the six fixed points coexist $(r=11, s=9)$.

Table 1. The nondegenerate critical points $\xi^{*}$ and their instability index $k: \xi_{0_{1}}^{*}$ and $\xi_{\pi_{1}}^{*}$ are the only nonlinear centers and the rest of them are saddles.

\begin{tabular}{cccc}
\hline fixed points & parameters & eigenvalues & index $k$ \\
\hline$\xi_{0_{1}}^{*}, \xi_{\pi_{1}}^{*}$ & $r, s>0$ & $\lambda_{1}, \lambda_{2}, \lambda_{3}, \lambda_{4}>0$ & 0 \\
$\xi_{0_{2}}^{*}, \xi_{\pi_{2}}^{*}$ & $r<s$ & $\lambda_{1}<0, \lambda_{2}>0, \lambda_{3}<0, \lambda_{4}>0$ & 2 \\
$\xi_{0,}^{*}, \xi_{\pi_{3}}^{*}$ & $3 \sqrt[3]{\frac{r^{2}}{4}}-1<s<r$ & $\lambda_{1}, \lambda_{2}, \lambda_{3}<0, \lambda_{4}>0$ & 3 \\
\hline
\end{tabular}

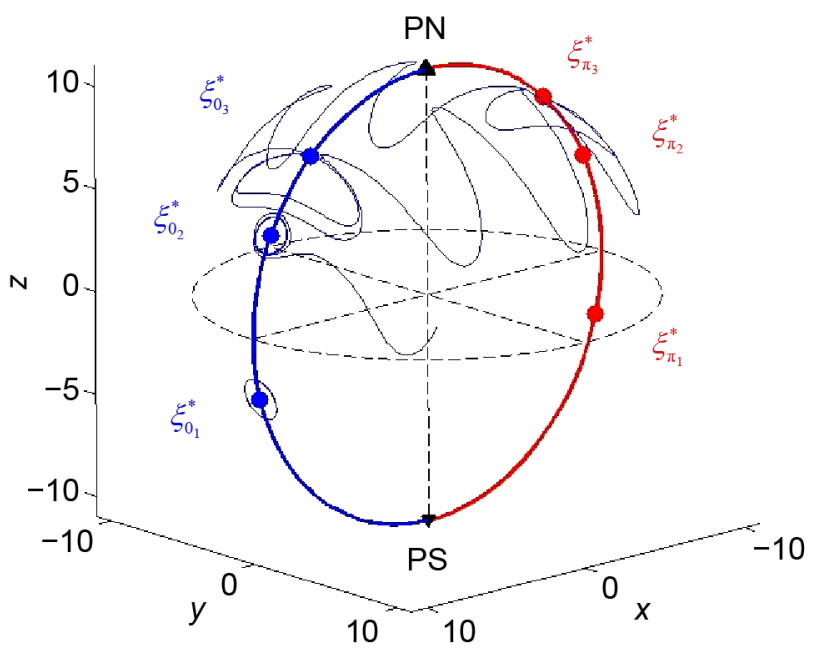

Figure 4. The $\left[\theta^{*}, \varphi^{*}\right]$ components of six fixed points $\xi^{*}$ in the orange-zone of Figure 3, represented in the dimensionless Bloch sphere, for $r=11, s=9$. Blue: the arc $\theta=0$, red: the arc $\theta=\pi$. The projection of trajectories, starting at an initial condition close to each $\xi^{*}$, is also plotted (only for $\xi_{0_{j}}^{*}$, for symmetry and clarity reasons) displaying the local behavior, according to the corresponding index $k$ (we use $\varepsilon=20$ ). The degenerate points, PN and PS, are plotted in black. 


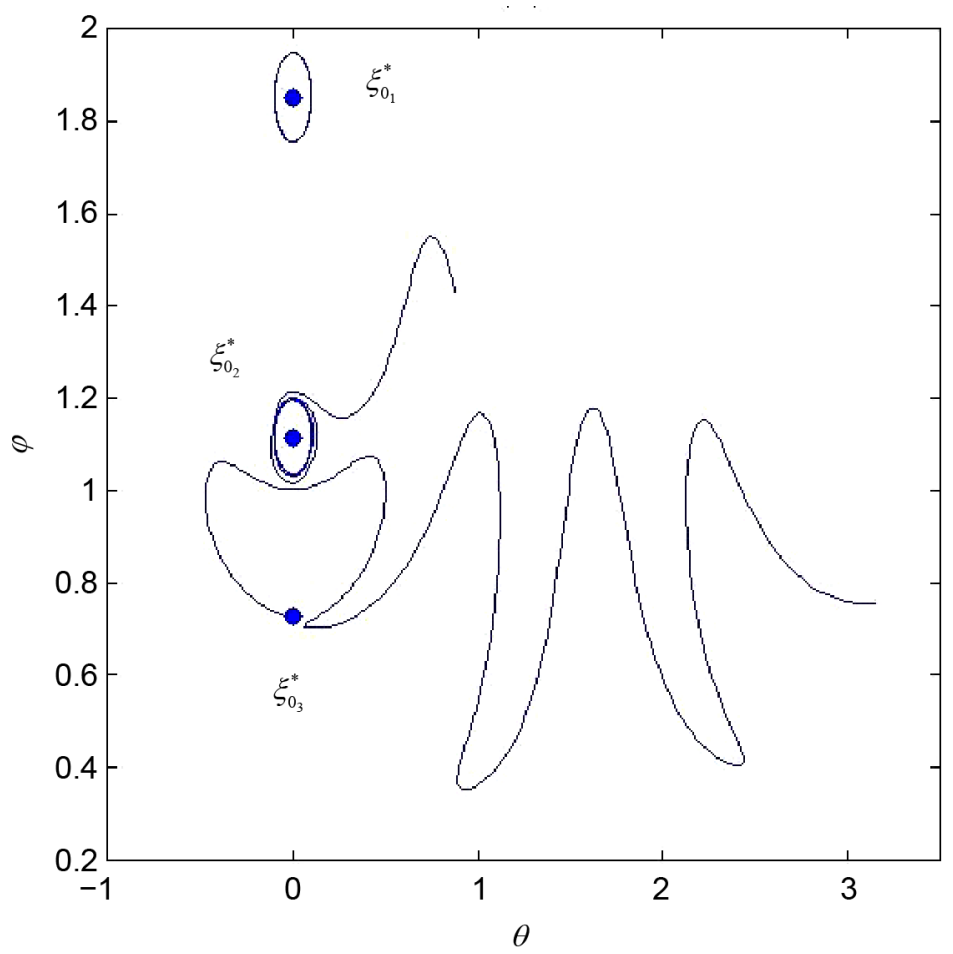

(a)

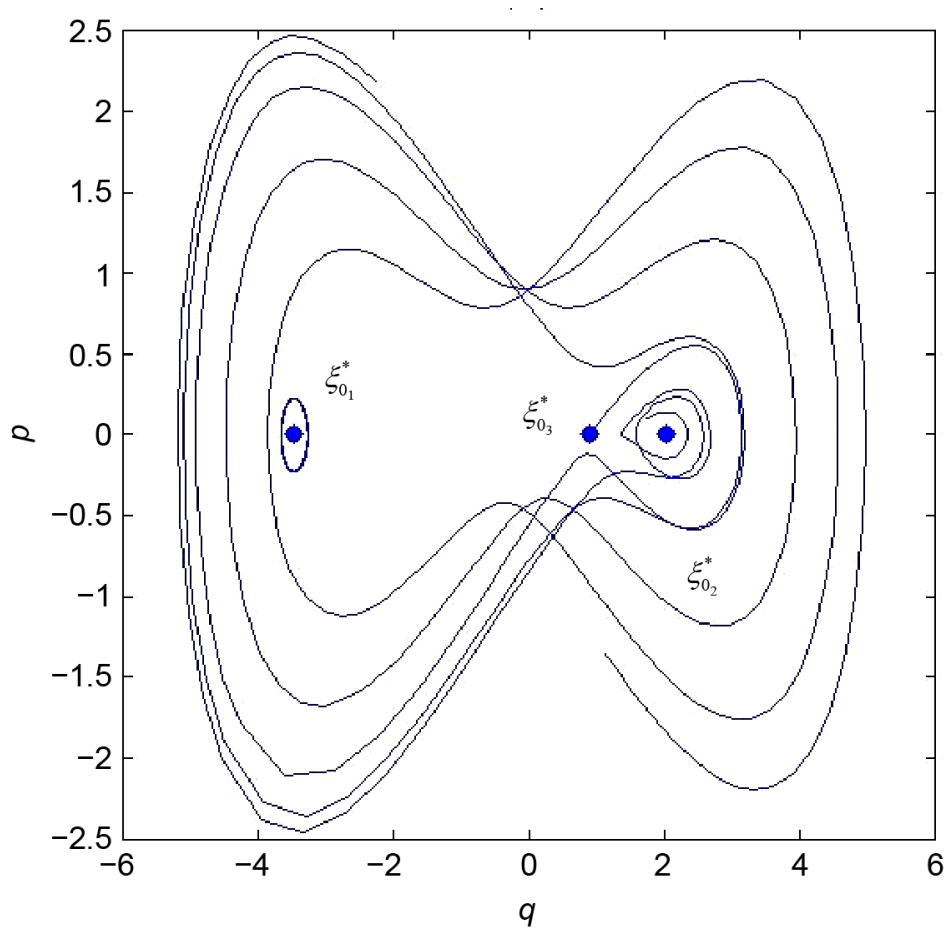

(b)

Figure 5. (a) The $\left[\theta^{*}, \varphi^{*}\right]$-components of $\xi_{0_{j}}^{*}, j=1,2,3$ of the Figure 4, represented in the quantum $(\theta, \varphi)$-phase space. The projection of nearby orbits is also plotted. (b) The $\left[q^{*}, p^{*}\right]$-components of the same $\xi_{0_{j}}^{*}$, represented in the classical $(q, p)$-phase space. 


\subsection{Note}

The other two fixed points of the system, $[0,0, \pm r, 0,0]$, which are not described by the change of variables $(\xi=[\theta, \varphi, q, p])$, result degenerate critical points of the energy $\langle h\rangle$ of Equation (17) as a function of $[x, y, z, q, p]$, since they force the vanishing of the Hessian matrix of $\langle h\rangle$.

\section{Conclusions}

The dimensionless formulation of Equations (2)-(6), given by Equations (11)-(15), allows for reduction in the number of system's parameters to three: $r$, $s$, and $\varepsilon$. The posterior change of variables (CV) has additional advantages as highlighted before: the reduction of the system's dimension through proper consideration of the uncertainty principle constraint. It also affords a convenient way to obtain the fixed points, providing a better characterization of them, according to the system's parameters, and depending only on the classical degree of freedom, $q$. We can display a relation between the new quantum degrees of freedom, $\theta$ and $\varphi$, which turn out to be canonically "spherical-conjugates", making the system's fixed points to be the critical points of the energy function $\langle h\rangle$. In addition, the CV illustrates the role of each parameter in the system dynamics in a very clear fashion: $\varepsilon$ can only play a role in the stability analysis, ensuring that the unstable fixed points are saddles. The parameter $s$ accounts for the range of possible values that $q$ can achieve at each fixed point, dividing the phase space into three regions. Finally, the parameter $r$ forces the uncertainty principle to play a fundamental role in the appearance/disappearance of fixed points, thus governing the presence of bifurcations in the $S U(2)$ nonlinear semiquantum dynamics.

The putative presence of chaotic dynamics in this system, for some $(r, s)$ parameter's region, is still under consideration and will be reported elsewhere.

Remark: by virtue of the $\mathrm{SU}(2)$ Lie algebra, the uncertainty principle becomes a constant of the motion. Thus, we claim that the methodology used in the present work applies even in the case in which the quantum subsystem of Equation (1) was nonlinear in the spin variables (as in the non-dissipative Hamiltonian case treated in [17], which can be used to model a SQUID). Despite the nonlinearity in the quantum subsystem, the uncertainty principle would remain there a constant of the motion. This and other topics related to semiquantum dynamics will be part of future work.

\section{Acknowledgements}

The authors would like to thank the reviewers for their suggestions to improve the readability of the paper. C. Sarris dedicates this work to Professor Araceli Proto, in memoriam. This work was partially supported by Programación Cientfica UBACyT 2014-2017 (20020130200093BA GEF), Ministerio de Educación, Argentina. 


\section{References}

[1] Porter, M.A. (2001) Nonadiabatic Dynamics in Semiquantal Physics. Reports on Progress in Physics, 64, 1165-1189. https://doi.org/10.1088/0034-4885/64/9/203

[2] Ballentine, L.E. (2001) Is Semi-Quantum Chaos Real? Physical Review E, 63, Article ID: 056204. https://doi.org/10.1103/PhysRevE.63.056204

[3] Cooper, F., Dawson, J.F., Meredith, D. and Shepard, H. (1994) Semiquantum Chaos. Physical Review Letters, 72, 1337-1340. https://doi.org/10.1103/PhysRevLett.72.1337

[4] Blumel, R. and Esser, B. (1994) Quantum Chaos in the Born-Oppenheimer Approximation. Physical Review Letters, 72, 3658-3661. https://doi.org/10.1103/PhysRevLett.72.3658

[5] Kowalski, A.M., Plastino, A. and Proto, A.N. (2002) Classical Limits. Physics Letters $A$, 297, 162-172. https://doi.org/10.1016/S0375-9601(02)00034-8

[6] Sarris, C.M. and Proto, A.N. (2009) Information Entropy and Nonlinear Semiquantum Dynamics. International Journal of Bifurcation and Chaos, 19, 3473-3484. https://doi.org/10.1142/S0218127409024918

[7] Ma, J. and Yuan, R.K. (1997) Semiquantum Chaos. Journal of the Physical Society of Japan, 66, 2302-2307. https://doi.org/10.1143/JPSJ.66.2302

[8] Goldstein, H. (1950) Classical Mechanics. Addison-Wesley, Reading, MA.

[9] Bonci, L., Roncaglia, R., West, B.W. and Grigolini, P. (1992) Semiclassical Chaos, the Uncertainty Principle, and Quantum Dissipation. Physical Review A, 45, 8490-8500. https://doi.org/10.1103/PhysRevA.45.8490

[10] Schanz, H. and Esser, B. (1997) Mixed Quantum-Classical versus Full Quantum Dynamics: Coupled Quasiparticle-oscillator System. Physical Review A, 55, 3375-3387. https://doi.org/10.1103/PhysRevA.55.3375

[11] Sarris, C.M., Caram, F. and Proto, A.N. (2004) The Uncertainty Principle as Invariant Motion for Time-dependent Hamiltonians. Physics Letters A, 324, 1-8. https://doi.org/10.1016/j.physleta.2004.02.036

[12] Alhassid, Y. and Levine, R.D. (1977) Entropy and Chemical Change. III. The Maximal Entropy (Subject to Constraints) Procedure as a Dynamical Theory. The Journal of Chemical Physics, 67, 4321-4400. https://doi.org/10.1063/1.434578

[13] Kowalski, A.M., Plastino, A. and Proto, A.N. (1997) A Semiclassical Statistical Model for Quantum Dissipation. Physica A, 236, 429-447. https://doi.org/10.1016/S0378-4371(96)00379-2

[14] Jaynes, E.T. (1957) Information Theory and Statistical Mechanics. Physical Review, 106, 620. https://doi.org/10.1103/PhysRev.106.620

[15] Strogatz, S.H. (2000) Nonlinear Dynamics and Chaos with Applications to Physics, Biology, Chemistry and Engineering. Perseus Publishing, Cambridge.

[16] Velhurst, F. (1996) Nonlinear Differential Equations and Dynamical Systems. 2nd Ed., Springer-Verlag, Berlin, Heidelberg, New York.

[17] Bonilla, L.L. and Guinea, F. (1992) Collapse of the Wave Packet and Chaos in a Model with Classical and Quantum Degrees of Freedom. Physical Review A, 45, 7718-7728. https://doi.org/10.1103/PhysRevA.45.7718 